British Journal of Psychiatry (1994), 164, 123-134

\title{
Correspondence
}

Contents: Lithium neurotoxicity at subtherapeutic serum levels/Antidepressant effects of ECT/Thalamofrontal psychosis/Parental bonding/Neural network model of amnesia/Suicide prevention/Seasonal affective disorder/Late-onset schizophrenia versus late paraphrenia/Benzodiazepine fatal poisonings/ Attachment theory/Variations on the theme of euthanasia/DIDMOAD (Wolfram) syndrome/Cognitive impairment and clozapine/Lithium dosage and inositol levels.

\section{Lithium neurotoxicity at subtherapeutic serum levels}

SIR: I read with interest the report by Bell et al (BJP, May 1993, 162, 689-692) reporting four cases of lithium neurotoxicity at normal therapeutic levels. I would like to report a case in which a reversible neurotoxic syndrome was associated with the administration of lithium carbonate, at subtherapeutic levels, and concurrent neuroleptic medication.

Case report. The patient, a 38-year-old man with idiopathic epilepsy (controlled on carbamazepine) and a chronic psychotic disorder, had proved unresponsive to conventional neuroleptic medication. On the basis of a previously satisfactory response to lithium, he was commenced on lithium carbonate (Priadel, $800 \mathrm{mg}$ daily) in addition to oral (haloperidol and chlorpromazine) and depot (fluphenazine) neuroleptics which had previously been tolerated well with only mild Parkinsonian side-effects. Eighteen days later the patient had a generalised seizure and over the following days exhibited increasingly prominent ataxia and myoclonus in all limbs. Various investigations performed at this time including a cranial computerised tomographic scan were within normal limits apart from an elevated serum alkaline phosphatase (184 IU/1). A serum lithium level ( 12 hours post-dose) 12 days following commencement of lithium had been $0.55 \mathrm{mmol} / 1$. Lithium was subsequently discontinued and the patient's neurological status reverted to normal.

Three months later lithium carbonate was prescribed again, this time at a dose of $\mathbf{4 0 0} \mathrm{mg}$ daily. Four days later a similar pattern of neurological abnormalities were observed whereupon further doses of lithium carbonate were withheld. A serum lithium level 10 hours following the last dose of lithium carbonate was $0.18 \mathrm{mmol} / \mathrm{l}$. Within 72 hours the neurological disturbance had settled completely.

The patient was rechallenged with lithium carbonate on a third occasion a month later at a dose of $\mathbf{4 0 0} \mathrm{mg}$ daily. Three days later ataxia and myoclonus were again observed with a serum lithium level (11 hours post-dose) of $0.25 \mathrm{mmol} / \mathrm{l}$. Five days later these abnormalities had again disappeared.

Debate persists concerning the hazards of neurotoxic sequelae in patients receiving concurrent treatment with lithium and neuroleptics; evidence from systematic studies (Baastrup et al, 1976; Goldney \& Spence, 1984) having failed to confirm any such interactions. This case, despite the presence of some atypical features, suggests that in some circumstances serious neurotoxic sequelae may accompany lithium therapy, even where serum levels are below the usual therapeutic range.

Banstrup, P. C., Hollnagel, P., Sorensen, R., et al (1976) Adverse reactions in treatment with lithium carbonate and haloperidol. JAMA, 236, 2645-2646.

GOLDNEY, R. D. \& SPENCE, N. D. (1986) Safety of the combination of lithium and neuroleptic drugs. American Journal of Psychiatry, $143,882-884$.

Floor B, Medical School

Richard Prettyman

Queen's Medical Centre

Nottingham NG7 2UH

\section{Antidepressant effects of ECT}

SIR: In their thoughtful review on the onset and rate of antidepressant effects of electroconvulsive therapy (ECT), Drs Scott \& Whalley (BJP, June 1993, 162, 725-732) point to several major lacunae in the current knowledge. We wish to supplement the following on the subject.

On the question of ECT in drug-naive, depressed patients, it has been shown that, at least in endogenous depressive patients, the antidepressant effect of ECT is faster than that of imipramine (Gangadhar et al, $B J P$, September 1982, 141, 267-371). Delayed onset of antidepressant effect of ECT is uncertain. A lag in the onset of therapeutic effect is indicated by the study of Jagadeesh et al (1992). They showed that in drug-naive, endogenous depressive patients, a single, bilateral, sinewave ECT produced timedependent therapeutic effects by two weeks when the magnitude of this effect was comparable to that produced by the conventional alternate-day ECTs. 
The frequency of ECT remains largely a matter of tradition, with little consistency in the literature. We (Gangadhar et al, 1993) have also found that twiceweekly bilateral ECT was comparable to a thriceweekly schedule for melancholic depressive patients, half of whom were drug-naïve. The depression scores reduced comparably in a given timeframe, despite the twice-weekly ECT group receiving significantly fewer ECTs. ECTs given twice a week had the advantage of obtaining optimal seizure duration in all ECT sessions (Janakiramaiah et al, 1992).

The matter is somewhat unsettled once again by Kellner et al (1992). They demonstrated a more rapid antidepressant effect with bilateral ECT, three times a week, than with once-a-week bilateral ECT in geriatric depressives.

Until the heterogeneity of patient characteristics, type of depression and prior medication are taken into account, studies of the onset as well as rate of improvement with ECT cannot be expected to yield consistent conclusions. The suggestion by Drs Scott \& Whalley to examine different schedules of ECT in a large population of depressives is hence especially timely. So also is the need to incorporate more sensitive techniques to monitor even smaller, early changes that may be induced by ECT in depression.

Gangaibhar, B. N., Janakiramaiah, N., Subbakrishna, D. K., et al (1993) Twice versus thrice weekly ECT in melancholia: a double blind prospective comparison. Journal of Affective Disorders, 27, 273-278.

Jagiadersh, H. N., Gangadhar, B. N., Janakiramaiah, N., et al (1992) Time dependent therapeutic effect of single electroconvulsive therapy (ECT) in endogenous depression. Journal of Affective Disorders. 24, $291-296$.

Janakiramaiah, N., Jyoti RaO, K. M.. Praveien, J., el al (1992) Seizure duration over ECT sessions: influence of spacing ECTs. Indian Journal of Psychiatry, 34, 124 127

Kellaner. C. H., Monrot, R. R., Pritchett, J.. el al (1992) Weekly ECT in geriatric depression. Convulsive Therapy, 8, 245253.

B. N. Gangadhar

N. JANAKIRAMAIAH

National Institute of

Mental Health and Neuro Sciences

Bangalore-560029, India

\section{Thalamo-frontal psychosis}

SIR: The suggested association by McGilchrist et al (BJP, July 1993, 163, 113-115) between thalamic lesions and a cyclical psychosis is intriguing. The authors correctly assert that theirs is the first clear report of cyclical psychosis following thalamic infarction. A careful reading of the literature may allow further insights into the possible significance of the case.
A total of 34 cases (McGilchrist et al, 1993; Starkstein et al, 1988a; Trimble \& Cummings, BJP, January $1981,138,56-59)$ of brain injury leading to mania have been reported. Fifteen of these cases had diencephalic lesions, eight frontal lobe, seven temporal lobe and the remaining four had widespread lesions. Twenty-one of the 34 had right-sided lesions, ten bilateral and three had isolated left-sided lesions. Six cases had evidence of damage in the thalamus, five of which were right and one bilateral (McGilchrist et al, 1993); none was left-sided. Two other cases had right thalamic and brainstem lesions.

The second of Trimble \& Cummings' two cases had a cyclical psychosis and a right thalamic arteriovenous malfunction which had bled into the ventricles (in addition, a lucency in the upper brainstem had been noted and at the time more significance was attributed to this aspect of the case). Six to 12 months after the intraventricular haemorrhage, this patient developed a manic-depressive-like illness which deteriorated gradually over the next five years into a schizophreniform state. Almost all the cases have lesions of the frontal lobe or its connections (Starkstein et al, 1988a). In addition, most of the lesions are right-sided.

Starkstein et al (1988b) addressed the specific role of basal ganglia and thalamus in different mood changes. They found a strong association between basal ganglia (more commonly the left and more specifically the caudate head) and post-stroke depression in contrast to the thalamus. They explain this finding on the basis of the anatomy of the biogenic amine pathways. The biogenic amine pathways run through the basal ganglia but are ventral to the thalamus. Starkstein et al have thus argued that they are spared in thalamic lesions and involved in basal ganglionic lesions. However, this hypothesis does not explain the association of euphoria and mania secondary to thalamic damage.

As we have pointed out earlier, it is a right-sided thalamic lesion which is associated with secondary mania. Oke et al (1978) have demonstrated lateralisation of norepinephrine (NE) in the human thalamus. The ventral tier, centromedian and parafascicular nuclei of the right thalamus contain much greater NE than do those areas on the left (Oke et al, 1978). Starkstein et al $(1988 a)$ have proposed that the induction of receptor supersensitivity by mesocorticolimbic dopaminergic or NE pathway lesions, or both, may mediate the neurobehavioural expression of mania. It is thus possible to explain the association of mania and right thalamic lesions on the basis of laterality of NE distribution in the thalamus.

Of course, the current state of knowledge does not permit a comprehensive explanation of mood 